\title{
The Positive Effect of LuoHanGuo as Sugar Substitute on Blood Glucose and Metabolism in Streptozotocin-Induced Diabetic Mice
}

\author{
Yoon Jeong Lee ${ }^{1,2, \dagger}$, Jain Jeong ${ }^{3, \dagger}$, Myoung Ok Kim ${ }^{4, *}$, Ju-Ock Nam ${ }^{5, *}$ \\ ${ }^{1}$ Department of Life Sciences, Yeungnam University, Gyeongsan 38541, Korea \\ ${ }^{2}$ Department of Optometry \& Vision Science, Kyungwoon University, Gumi 39160, Korea \\ ${ }^{3}$ School of Life Sciences, KNU Creative BioResearch Group (BK21 Plus Project), Kyungpook National University, Daegu 41566, Korea \\ ${ }^{4}$ Department of Animal Science, Kyungpook National University, Sangju 37224, Korea \\ ${ }^{5}$ School of Food Science \& Biotechnology, Kyungpook National University, Daegu 41566, Korea
}

\author{
${ }^{\dagger}$ These authors contributed equally \\ to this work as co-first authors. \\ *Correspondence to: \\ Nam JO, \\ Tel: +82-53-950-7761 \\ Fax: +82-53-950-7762 \\ E-mail: namjo@knu.ac.kr \\ Kim $\mathrm{MO}$, \\ Tel: +82-54-530-1234 \\ Fax: +82-54-530-1229 \\ E-mail: ok4325@knu.ac.kr \\ Received June 6, 2016 \\ Revised September 6, 2016 \\ Accepted September 12, 2016
}

\begin{abstract}
This study intended to compare the effects of the sugar-Omija extract tea (SO) and LuoHanGuo-Omija extract tea (LO) to blood glucose levels and metabolism among streptozotocin (STZ)-induced diabetic mice and to prove the positive effects of LuoHanGuo extract as a sugar substitute. According to the results, the rate of blood glucose increase and the blood glucose level was reduced, and the weight was increased. Aspartate aminotransferase (AST), alanine aminotransferase (ALT), triglyceride, low density lipoprotein cholesterol, and total cholesterol was decreased, and albumin, total protein, and high density lipoprotein cholesterol (HDL-C) was increased in the STZ/LO group as compared to the STZ group. Compared to the STZ/SO group, the rate of blood glucose increase and glucose tolerance was decreased. AST, ALT, and total cholesterol was decreased, and HDL-C level was high. In conclusion, the LO displayed hypoglycemic effect, prevented weight loss caused by diabetes, recovered liver function, and improved lipid metabolism. In addition, such positive effects were obviously shown during comparison with the experimental group treated with the SO. Therefore, the LO were considered as favorable food supplements that can be safely taken by persons with diabetes, obesity, hypertension, and heart disease; they have to control their sugar intake as well as their diet.
\end{abstract}

Key Words: LuoHanGuo, Omija, Sugar, Diabetes, Metabolism

\section{INTRODUCTION}

Diabetes is an endocrine disease that occurs due to abnormal function of insulin owing to increased insulin insufficiency or resistance. Along with genetic factor, diabetes is influenced by congenital factors (obesity), diet, insufficient exercise, and stress. Prolonged diabetes with hyperglycemia and abnormal lipid metabolism causes serious chronic complications such as vasculopathy like arteriosclerosis, hypertension, myocardial infarction, renal disease, neuropathy, retinopathy, and cataract (Soedamah-Muthu et al., 2006; West et al., 1983).
Excessive sugar intake is one of the risk factors associated with type 2 diabetes mellitus (T2DM) and insulin resistance: generally considered causing an increase risk of T2DM, whereas type 1 diabetes mellitus (T1DM) is considered an autoimmune disease that directly involved in the destruction of beta-cell (Steven et al., 2006). When it comes to dietary management of diabetic patients, sugar must be seriously controlled in order to prevent significant increase in blood glucose. Therefore, the purpose of diabetes treatment is to control not only the fasting blood glucose but also the postprandial glucose; their levels should be maintained

(a) This is an open-access article distributed under the terms of the Creative Commons Attribution Non-Commercial License (http://creativecommons.org/licenses/by-nc/4.0) which permits unrestricted noncommercial use, distribution, and reproduction in any medium, provided the original work is properly cited.

Copyrights @ 2016 by Korean Society of Microscopy 
as normally as possible to prevent and delay possible complications (Haller, 1998; Jenkins et al., 1988; Lebovitz, 1998). It is recommended for diabetic patients to take a small amount of food during each meal and have snacks in between in order to maintain blood glucose level within short- and long-term permissible range. However, the accessible snacks are processed foods with a lot of sugar content and these increase blood glucose more significantly than regular meals; therefore, certain snacks can worsen diabetes (Park et al., 2002; Shin, 2010).

Sugar is a natural sweetener that has been widely used for a long time throughout the world. Due to increased income and availability of processed foods, its consumption likewise increased (Bray et al., 2004). Aside from having bad effects on people's health and being a carcinogenic, sugar may result to deteriorated immunity, glucose homeostasis, and diabetes. The public is now aware of the harmful effects of sugar so there is a tendency to reduce its use and intake. Accordingly, many attempts have been made to search, discover, develop, and use natural sweeteners as sugar substitutes (Alonso \& Setser, 1994). Accordingly, it is necessary to discover natural food or natural functional food that does not cause radical blood glucose increase after intake, has low calories, has sweet taste, does not have side-effects during long-term use for diabetes treatment, and prevents diabetes complication. There is also a need to prove such food's effects (Alonso \& Setser, 1994; Park \& Han, 2015; Park et al., 2015).

LuoHanGuo (Momordicae grosvenori swingla) is a perennial herb from the Cucurbitaceae family. It is a special species that grows only in Guilin of the southern Guangxi Province in China (Huang, 2006; Institute of Beijing Botany of Academic Sinica, 1975; Li \& Zhang, 2000). It has been used in China as an excellent traditional folk treatment for respiratory, cardiovascular, and digestive diseases (Tang et al., 2007; Zhou \& Tang, 2006). Physiologically, its various effects (anticancer, antioxidant, anti-inflammatory, and anti-allergic activities) have been scientifically proven (Chen et al., 2007; Takasaki et al., 2003; Ukiya et al., 2002a, 2002b). It has been reported to be particularly effective in treating diseases that require moderate sugar intake such as diabetes, obesity, hypertension, and heart disease (Si et al., 1996; Suzuki et al., 2005; Takeo et al., 2002). The glycoside of LuoHanGuo did not originate from saccharide but rather, from the glycoside of triterpene. Particularly, triterpenoid glycoside V (mogroside V) is known to be an important component of sweetener (Yoshikawa et al., 2005). It has evenly high content and sugar content among the fruits of LuoHanGuo. Moreover, it is approximately 300 400 times sweeter than regular sugar (Hussain et al., 1990; Suzuki et al., 2005). However, it does not contain any calories and is not absorbed in the body. It does not cause glycometabolism, thus, it is a perfect sugar substitute for diabetics, obese people, and those who are on a diet (Chen et al., 2007; Si et al., 1996).
Today, sugar is mainly used for Omija-containing food and beverages in Korea. Omija (Schizandra chinensis) is known to be a health functional food. It has been reported to beneficial effects to health with anti-oxidant, and anti-diabetic properties (Kim \& Choi, 2008; Kim et al., 2009). However, sugar contained such food and beverages is somewhat avoided among diabetics, obese people, and those who are on a diet. For this reason, the research team of this study developed and produced the LuoHanGuo-Omija extract tea (LO) in recognition of the necessity to develop Omija-containing food and beverages using LuoHanGuo as a sugar substitute. Therefore, in this study, we investigated the possibility and utility that our developed LuoHanGuo-based-sweetenerOmija extract (LO) as a health functional food compare to sugar-based extract (sugar-Omija extract tea, $\mathrm{SO}$ ) in streptozotocin (STZ)-induced diabetic mouse model; reveling the hyperglycemic condition. Moreover, it aimed to prove the positive effects of LuoHanGuo extract as a sugar substitute. Additionally, we also determine whether our developed LO by itself affected improving diabetic condition in diabetic mouse model.

\section{MATERIALS AND METHODS}

\section{Animals and Reagents}

A total of 30 C57BL/6 mice that were seven weeks old were purchased from Hyochang Bio (Korea) and used for the experiment. All experimental animals were kept under controlled environment with 12 hours of lighting cycle, $22^{\circ} \mathrm{C} \pm 1^{\circ} \mathrm{C}$ of temperature, and $50 \% \pm 4 \%$ of relative humidity. Sterilized feed (Feedlab, Korea) and water were freely fed for the experimental mice's adaptation to the environment. All reagents used including STZ (N-(Methylnitrosocarbamoyl)$\alpha$-D-glucosamine) were purchased from Sigma-Aldrich Inc. (USA). The SO (patent application No. 2013-0044118) and LO (patent application No. 10-2016-0063508) that were developed, manufactured, and sold by the Pureunsan Cham Farm Co., Ltd. (Korea) and Industry-Academic Cooperation Foundation of Kyungwoon University (Korea) were used for the experiment.

\section{Diabetes Induction and Omija Extract Tea Administration}

For diabetes induction, eight-week old male C57Bl/6J mice were used. STZ (Sigma-Aldrich) was dissolved in $0.1 \mathrm{M}$ citrate solution ( $\mathrm{pH} 4.5$ ). About $150 \mathrm{mg}$ per $\mathrm{kg}$ of body weight was administered each time through intraperitoneal injection. Three days after the STZ injection, tail vein blood was taken using a blood glucose monitoring device (Accu-Chek Go Kit [model GS]; Roche, Germany). Through this method, the mice with non-fasting blood glucose level of more than 250 $\mathrm{mg} / \mathrm{dL}$ were identified as diabetes-induced mice and these 
were used in the experiment.

In order to select the experimental group, a total of six groups with five mice per group were classified to conduct the experiment as follows: 1) water-treated non-diabetic group (normal group, $\mathrm{n}=5$ ); 2) SO-treated non-diabetic group (SO group, $\mathrm{n}=5$ ); 3) LO-treated non-diabetic group (LO group, $\mathrm{n}=5$ ); 4) water-treated diabetic group (STZ group, $\mathrm{n}=5$ ); 5) SO-treated diabetic group (STZ/SO group, $\mathrm{n}=5)$; and 6) LOtreated diabetic group (STZ/LO group, $n=5$ ) (Table 1). Based on the actual average intake of a typical man, each group was orally administered with $250 \mu \mathrm{L}$ of water, SO, and LO once a day for two weeks. All procedures of the animal testing were approved by the Kyungpook National University Industry Foundation (protocol \# 2015-0036).

\section{Measurement of Blood Glucose and Body Weight}

In order to observe the glucose tolerance depending on administered testing material, the Intraperitoneal Glucose Tolerance Tests (IPGTTs) were conducted. By using mice $(n=5)$ on the 10th day of receiving each testing material, glucose was intraperitoneally injected at a concentration of 1.5 $\mathrm{g} / \mathrm{kg}$ of body weight after six hours of fasting. By using a blood glucose monitoring device, the blood-glucose concentration of the tail vein blood was measured at $0,30,60,90$, and 120 minutes interval. In addition, non-fasting blood glucose from the tail vein blood was measured using a blood glucose monitoring device and the weight of each mouse was assessed every two days during the period of administering the tests.

\section{Hematological Analysis}

Serum biochemical analysis was conducted to assess the liver, kidney, and lipid metabolism. After two weeks of administering the testing materials, the mice were anesthetized with Avertin (Sigma Aldrich, USA) and their blood was collected through retro orbital blood collection. The gathered blood samples were stored in room temperature for about 30 minutes and then the serum was separated through centrifugation for 15 minutes at $4^{\circ} \mathrm{C}$. Through this method, analysis of the physiological and metabolic parameters on a total of 12 items was requested to KNOTUS

Table 1. Classification of experimental groups

\begin{tabular}{lcl}
\hline \multicolumn{1}{c}{ Group } & $\mathrm{n}$ & \multicolumn{1}{c}{ Classification } \\
\hline Non-STZ treated group & \\
Normal & 5 & No/water \\
SO & 5 & No/sugar-Omija extract tea \\
LO & 5 & No/LuoHanGuo-Omija extract tea \\
STZ treated group & & \\
STZ & 5 & Streptozotocin/water \\
STZ/SO & 5 & Streptozotocin/sugar-Omija extract tea \\
STZ/LO & 5 & Streptozotocin/LuoHanGuo-Omija extract tea \\
\hline
\end{tabular}

Co., Ltd. (formerly Korea Animal Medical Science Institute). The indexes analyzed from the separated serum were the following (Table 2).

\section{Histological Observation}

In order to observe general changes in the removed pancreas, histological examination was conducted. The removed pancreas was treated by placing it in $10 \%$ phosphate buffered formalin (neutral) and then cleansed with running water. The alcohol concentration was gradually increased to facilitate dehydration. After removing extra alcohol with xylene, the pancreas was embedded. The produced block was section in 4 5 $\mu \mathrm{m}$ thickness using a microtome (model 45; Lipshaw, USA) to make a slide. After dyeing it with H\&E and stitched, it was observed with an optical microscope (BH-2; Olympus, Japan).

\section{Statistical Method}

All experimental results were analyzed using average and standard error (mean \pm SE). The data were analyzed using SPSS for Windows ver. 14.0 (SPSS Inc., USA). To verify statistical significance, Student's t-test was conducted. Also, $\mathrm{p}$-value less than $\mathrm{p}<0.05$ was considered as significant value.

\section{RESULTS}

\section{Measurement of Blood Glucose}

The results of blood glucose measurement are shown in Fig. 1 and 2. In the non-STZ treated group, the concentration of blood glucose tend to show similar pattern during the experimental period (Fig. 1). As shown in Fig. 2, the concentration of blood glucose in the STZ treated group increased during the experimental period after inducing diabetes with STZ. Regarding the rate in increase of blood glucose, the STZ/SO group was lower than the STZ group whereas the STZ/LO group showed lower values than that of the STZ/SO group. From the third day of administering the

Table 2. Biochemical parameter of serum

\begin{tabular}{ll}
\hline \multicolumn{1}{c}{ Function } & \multicolumn{1}{c}{ Parameter } \\
\hline Liver & AST, aspartate aminotransferase \\
& ALT, alanine aminotransferase \\
& ALP, alkaline phosphatase \\
& Albumin \\
& T. protein, total protein \\
& T. bilirubin, total bilirubin \\
& BUN, blood urea nitrogen \\
& Creatinine \\
Kidney & TG, triglyceride \\
& HDL-C, high density lipoprotein cholesterol \\
& LDL-C, low density lipoprotein cholesterol \\
& T. cholesterol, total cholesterol \\
\hline
\end{tabular}




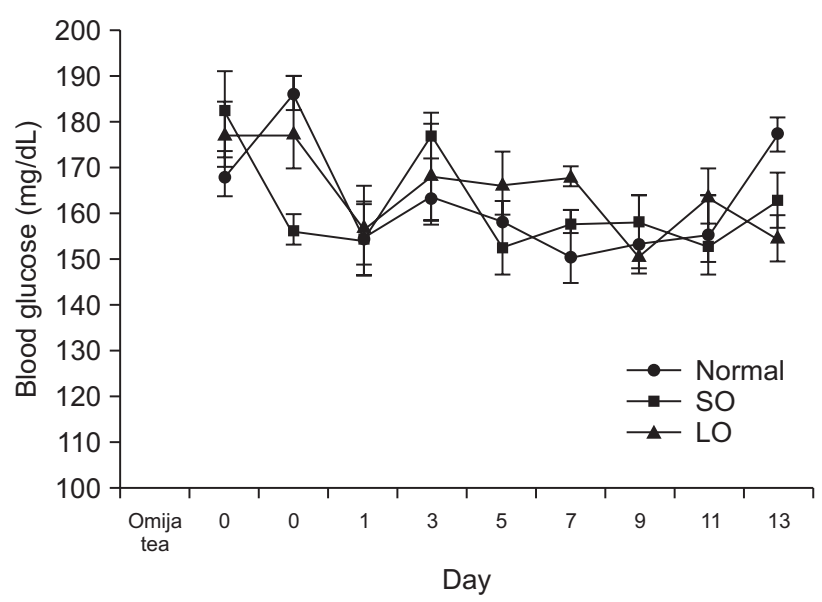

Fig. 1. Change of blood glucose after administration of Omija extract tea in non-streptozotocin treated mice. Statistical analysis was by a Student's t-test. All values are presented as mean \pm standard error. SO, sugar-Omija extract tea; LO, LuoHanGuo-Omija extract tea.

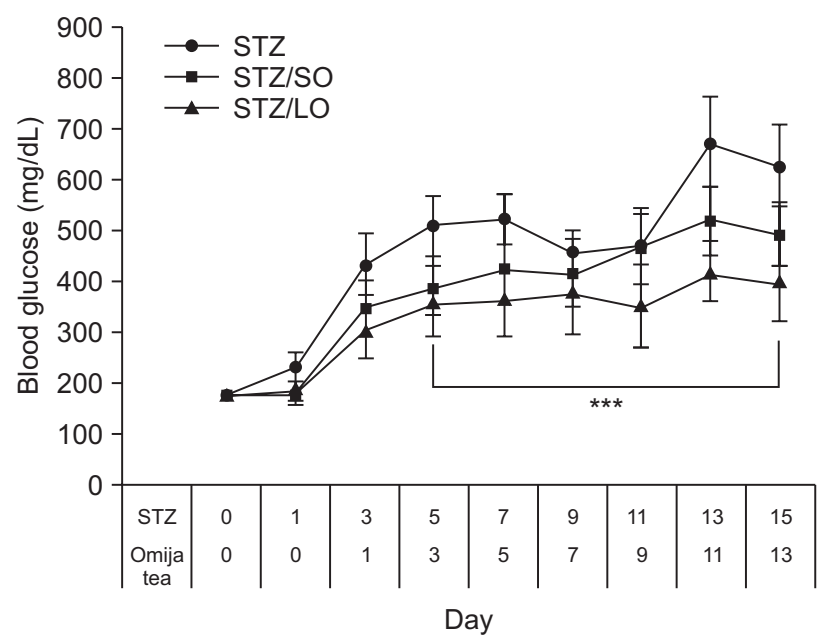

Fig. 2. Change of Blood glucose after administration of Omija extract tea in streptozotocin (STZ)-induced diabetic mice. Statistical analysis was by a Student's t-test. All values are presented as mean \pm standard error. SO, sugar-Omija extract tea; LO, LuoHanGuo-Omija extract tea. ***Significantly different from the STZ/SO group by t-test $(\mathrm{p}<0.001)$.

Omija extract after five days of the STZ treatment, the blood glucose in the STZ/LO group showed significantly lower values than that of the STZ group $(\mathrm{p}<0.001)$.

\section{Intraperitoneal Glucose Tolerance Tests}

The results of the IPGTTs are the following (Fig. 3). The glucose was administered at a concentration of $1.5 \mathrm{~g} / \mathrm{kg}$ and the mice's blood glucose level was measured at 30,60, 90, and 120 minutes interval. According to the results, the blood glucose of the STZ/LO group was relatively lower than that of the STZ group and STZ/SO group. The blood glucose of the tail vein blood was measured at 120 minutes with the following values: $520.7 \pm 7 \mathrm{mg} / \mathrm{dL}$ in the STZ group;

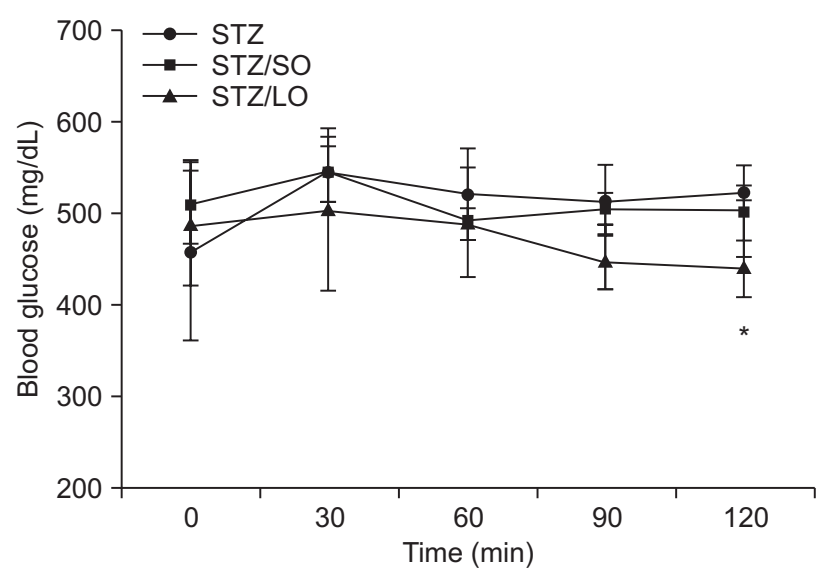

Fig. 3. Incremental Blood glucose after administration of Omija extract tea in streptozotocin (STZ)-induced diabetic mice. Statistical analysis was by a Student's t-test. All values are presented as mean \pm standard error. SO, sugar-Omija extract tea; LO, LuoHanGuo-Omija extract tea. *Significantly different from the STZ/SO group by t-test $(\mathrm{p}<0.05)$.

and $439.0 \pm 31 \mathrm{mg} / \mathrm{dL}$ in the STZ/LO group. Therefore, it significantly decreased in the STZ/LO group as compared to the STZ group $(\mathrm{p}<0.05)$.

\section{Measurement of Body Weight}

The results of weight measurement are shown in Fig. 4 and 5. The weight in the non-STZ treated group almost did not show fluctuation during the experimental period (Fig. 4). As shown in Fig. 5, the pattern of weight change in the STZ treated group was similar to the STZ/SO group and STZ/LO group, and from the third day of administering the Omija extract after five days of STZ treatment, the weight in the STZ/LO group significantly increased as compared to that of the STZ group $(\mathrm{p}<0.001)$.

\section{Hematological Analysis}

The results of the index analysis on functional change of each organ are shown in Tables 3 and 4 .

The results of the index analysis on functional change of each organ among the non-STZ treated group are shown in Table 3. There was no significant difference among the groups in terms of aspartate aminotransferase (AST), alanine aminotransferase (ALT), and total protein values in the liver function test. The alkaline phosphatase (ALP) in the SO group $(\mathrm{p}<0.001)$ and LO group $(\mathrm{p}<0.05)$ significantly decreased as compared to that of the normal group. Albumin in the LO group significantly increased as compared to that of the SO group $(\mathrm{p}<0.05)$. Total bilirubin of the LO group significantly increased compared to the normal group and SO group $(\mathrm{p}<0.05)$. Blood urea nitrogen $(\mathrm{BUN})$ of the renal function test significantly decreased in the SO group $(\mathrm{p}<0.05)$ and LO group $(\mathrm{p}<0.05)$ compared to the normal group. There was no significant difference among the groups in terms of 
creatinine level. Moreover, regarding lipid metabolism, there was no significant difference among the groups in terms of triglyceride, high density lipoprotein cholesterol (HDL-C), low density lipoprotein cholesterol (LDL-C), and total cholesterol.

The results of the index analysis on functional change of each organ among the STZ treated group are shown in Table 4 .

There was no significant difference among the groups in terms of ALP of the liver function test and total bilirubin. AST

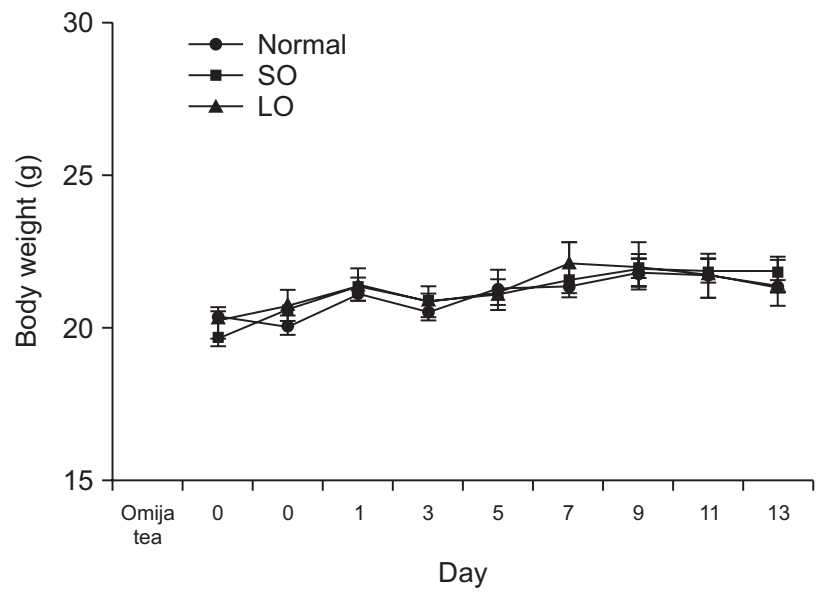

Fig. 4. Change of body weight after administration of Omija extract tea in non-streptozotocin treated mice. Statistical analysis was by a Student's t-test. All values are presented as mean \pm standard error. SO, sugar-Omija extract tea; LO, LuoHanGuo-Omija extract tea. in the STZ/SO group significantly increased compared to the STZ group $(\mathrm{p}<0.05)$. AST in the STZ/LO group decreased as compared to that of the STZ group and significantly decreased as compared to that of the STZ/SO group $(\mathrm{p}<0.001)$. ALT in the STZ/LO group decreased compared to the STZ group and significantly decreased as compared to that of the STZ/SO group ( $\mathrm{p}<0.001$ ). Albumin of the STZ/SO group significantly increased as compared to that of the STZ group $(\mathrm{p}<0.001)$

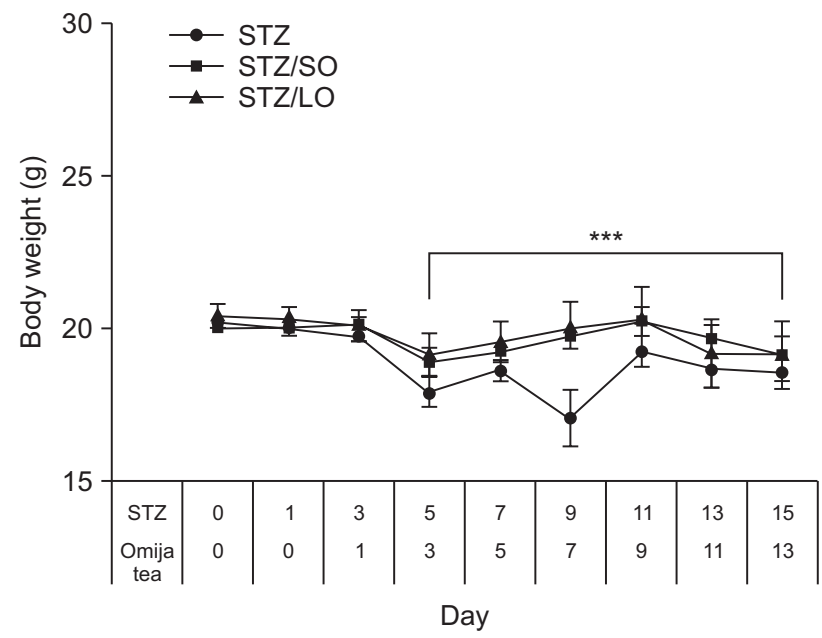

Fig. 5. Change of body weight after administration of Omija extract tea in streptozotocin (STZ)-induced diabetic mice. Statistical analysis was by a Student's t-test. All values are presented as mean \pm standard error. SO, sugar-Omija extract tea; LO, LuoHanGuo-Omija extract tea. ***Significantly different from the STZ/SO group by t-test $(\mathrm{p}<0.001)$.

Table 3. Change of parameters on liver, kidney, and lipid metabolism in non-STZ treated mice

\begin{tabular}{|c|c|c|c|c|}
\hline \multirow{2}{*}{\multicolumn{2}{|c|}{ Parameter }} & \multicolumn{3}{|c|}{ Non-STZ group } \\
\hline & & Normal & SO & LO \\
\hline \multirow[t]{6}{*}{ Liver } & AST (IU/L) & $92.16 \pm 12.28$ & $144.72 \pm 40.31$ & $165.72 \pm 45.10$ \\
\hline & ALT (IU/L) & $36.30 \pm 2.60$ & $33.40 \pm 4.45$ & $42.82 \pm 5.86$ \\
\hline & $\operatorname{ALP}(\mathrm{IU} / \mathrm{L})$ & $438.52 \pm 20.48$ & $342.62 \pm 17.53^{\text {aa }}$ & $385.70 \pm 17.40^{\mathrm{a}}$ \\
\hline & Albumin (g/dL) & $1.74 \pm 0.09$ & $1.76 \pm 0.07$ & $1.93 \pm 0.06^{\mathrm{b}}$ \\
\hline & Total protein (g/dL) & $5.50 \pm 0.30$ & $5.45 \pm 0.24$ & $6.01 \pm 0.28$ \\
\hline & Total bilirubin (mg/dL) & $0.09 \pm 0.01$ & $0.10 \pm 0.01$ & $0.14 \pm 0.02^{\mathrm{a}, \mathrm{b}}$ \\
\hline \multirow[t]{2}{*}{ Kidny } & BUN (mg/dL) & $23.27 \pm 1.11$ & $20.60 \pm 0.34^{\mathrm{a}}$ & $20.48 \pm 0.99^{\mathrm{a}}$ \\
\hline & Creatinine (mg/dL) & $0.45 \pm 0.01$ & $0.44 \pm 0.04$ & $0.49 \pm 0.03$ \\
\hline \multirow[t]{4}{*}{ Lipid metabolism } & Triglyceride (mg/dL) & $58.76 \pm 6.37$ & $64.16 \pm 10.25$ & $52.84 \pm 4.33$ \\
\hline & $\mathrm{HDL}-\mathrm{C}(\mathrm{mg} / \mathrm{dL})$ & $54.26 \pm 3.99$ & $52.46 \pm 2.17$ & $53.51 \pm 2.28$ \\
\hline & $\mathrm{LDL}-\mathrm{C}(\mathrm{mg} / \mathrm{dL})$ & $6.23 \pm 1.19$ & $6.40 \pm 0.58$ & $6.72 \pm 1.17$ \\
\hline & Total cholesterol (mg/dL) & $95.78 \pm 7.93$ & $98.22 \pm 2.91$ & $95.96 \pm 4.53$ \\
\hline
\end{tabular}

Statistical analysis was by a Student's t-test.

All values are presented as mean \pm standard error for mice in each group.

STZ, streptozotocin; SO, sugar-Omija extract tea; LO, LuoHanGuo-Omija extract tea; AST, aspartate aminotransferase; ALT, alanine aminotransferase; ALP, alkaline phosphatase; BUN, blood urea nitrogen; HDL-C, high density lipoprotein cholesterol; LDL-C, low density lipoprotein cholesterol.

${ }^{\mathrm{a}}$ Significantly different from the normal group by t-test $(\mathrm{p}<0.05)$.

${ }^{\text {aa }}$ Significantly different from the normal group by t-test $(\mathrm{p}<0.001)$

${ }^{\mathrm{b}}$ Significantly different from the SO group by t-test $(\mathrm{p}<0.05)$. 
Table 4. Change of parameters on liver, kidney, and lipid metabolism in STZ-induced diabetic mice

\begin{tabular}{|c|c|c|c|c|}
\hline \multirow{2}{*}{\multicolumn{2}{|c|}{ Parameter }} & \multicolumn{3}{|c|}{ STZ group } \\
\hline & & STZ & STZ/SO & STZ/LO \\
\hline \multirow[t]{6}{*}{ Liver } & AST (IU/L) & $130.56 \pm 58.39$ & $236.24 \pm 27.86^{\mathrm{a}}$ & $103.46 \pm 11.84^{\mathrm{bb}}$ \\
\hline & ALT (IU/L) & $77.84 \pm 34.81$ & $86.94 \pm 10.60$ & $48.36 \pm 4.84^{\mathrm{bb}}$ \\
\hline & ALP (IU/L) & $713.06 \pm 318.89$ & $716.52 \pm 64.01$ & $578.94 \pm 59.98$ \\
\hline & Albumin (g/dL) & $1.52 \pm 0.68$ & $1.77 \pm 0.06^{\text {aa }}$ & $1.58 \pm 0.06^{\mathrm{b}}$ \\
\hline & Total protein $(\mathrm{g} / \mathrm{dL})$ & $5.15 \pm 2.30$ & $5.81 \pm 0.24^{\mathrm{a}}$ & $5.37 \pm 0.08$ \\
\hline & Total bilirubin (mg/dL) & $0.09 \pm 0.04$ & $0.10 \pm 0.01$ & $0.10 \pm 0.01$ \\
\hline \multirow[t]{2}{*}{ Kidny } & $\mathrm{BUN}(\mathrm{mg} / \mathrm{dL})$ & $32.30 \pm 14.45$ & $26.09 \pm 1.46$ & $36.38 \pm 4.16^{\mathrm{b}}$ \\
\hline & Creatinine (mg/dL) & $0.53 \pm 0.24$ & $0.51 \pm 0.03$ & $0.50 \pm 0.40$ \\
\hline \multirow[t]{4}{*}{ Lipid metabolism } & Triglyceride (mg/dL) & $132.26 \pm 59.15$ & $148.00 \pm 19.24$ & $126.28 \pm 10.91$ \\
\hline & $\mathrm{HDL}-\mathrm{C}(\mathrm{mg} / \mathrm{dL})$ & $57.26 \pm 25.61$ & $49.28 \pm 2.35$ & $59.96 \pm 3.18^{\mathrm{b}}$ \\
\hline & LDL-C (mg/dL) & $6.46 \pm 0.44$ & $6.15 \pm 0.51$ & $5.99 \pm 2.68$ \\
\hline & Total cholesterol (mg/dL) & $107.56 \pm 48.10$ & $114.64 \pm 6.10$ & $95.88 \pm 2.82$ \\
\hline
\end{tabular}

Statistical analysis was by a Student's t-test.

All values are presented as mean \pm standard error for mice in each group.

STZ, streptozotocin; SO, sugar-Omija extract tea; LO, LuoHanGuo-Omija extract tea; AST, aspartate aminotransferase; ALT, alanine aminotransferase; ALP, alkaline phosphatase; BUN, blood urea nitrogen; HDL-C, high density lipoprotein cholesterol; LDL-C, low density lipoprotein cholesterol.

${ }^{\mathrm{a}}$ Significantly different from the STZ group by $\mathrm{t}$-test $(\mathrm{p}<0.05)$.

${ }^{\text {aa }}$ Significantly different from the STZ group by t-test $(\mathrm{p}<0.001)$.

${ }^{b}$ Significantly different from the STZ/SO group by t-test $(\mathrm{p}<0.05)$.

${ }^{\mathrm{bb}}$ Significantly different from the STZ/SO group by t-test $(\mathrm{p}<0.001)$.

and albumin of the STZ/LO group increased compared to the STZ group and significantly decreased compared to the STZ/SO group $(\mathrm{p}<0.05)$. The total protein of the STZ/LO group increased while the total protein of the STZ/SO group significantly increased compared to the STZ group $(\mathrm{p}<0.05)$. Lastly, the BUN of the STZ/LO group increased compared to the STZ group and significantly increased as compared to that of the STZ/SO group $(\mathrm{p}<0.05)$. There was no significant difference in creatinine among the groups.

Regarding lipid metabolism, triglyceride, HDL-C, LDL-C, and total cholesterol of the STZ/LO group showed lower values compared to the STZ group and STZ/SO group. Particularly, total cholesterol of the STZ/LO group was significantly lower compared to the STZ/SO group ( $<<0.05$ ). HDL-C in the STZ/ LO group was higher as compared to that of the STZ group and significantly higher compared to the STZ/SO group $(\mathrm{p}<0.05)$.

\section{Histological Observation}

The pancreatic tissues were examined with an optical microscope, and those of the non-STZ treated group showed normally preserved acinar cell and beta-cell in the islets of Langerhans (Fig. 6A-C). Among the STZ treated groups, damages in the beta-cell and big vacuolation were noticeably observed in the STZ group and STZ/SO group (Fig. 6D and E). Compared to the STZ group, the STZ/LO group showed relatively normal beta-cell and blood vessel structure and there was no vacuolation observed (Fig. 6F).

\section{DISCUSSION}

Regardless of great developments in modern science, the incidence of diabetes has steadily increased. According to the Korea National Health and Nutrition Examination Survey in 2014, which was conducted by the Korea Centers for Disease Control and Prevention and Ministry of Health and Welfare among adults 30 years old and above, one out of 10 adults was diagnosed with diabetes ( $11.1 \%$ incidence rate, $13.5 \%$ for men, $8.8 \%$ for women). The incidence rate of diabetes was maintained at approximately $9 \%$ in the past 10 years since 2005 and it has increased by about $1 \% \sim 2 \%$ annually, resulting to $11.0 \%$ incidence rate in 2013 and $10.2 \%$ in 2014 (Korean Diabetes Association \& Korea Centers for Disease Control and Prevention, 2012; Ministry of Health and Welfare, 2014). Moreover, sugar consumption and intake related to insulin resistance (Kraegen et al., 1991; Luo et al., 1995; Nakamura et al., 2001; Storlien et al., 1991) also increased due to high income levels and availability of processed foods (Bray et al., 2004). On the other hand, there is increased awareness among the public about the harmful effects of sugar; thus, there is a tendency to reduce the use and intake of sugar and many attempts have been made to search, discover, develop, and use natural sweeteners as sugar substitutes (Alonso \& Setser, 1994). 

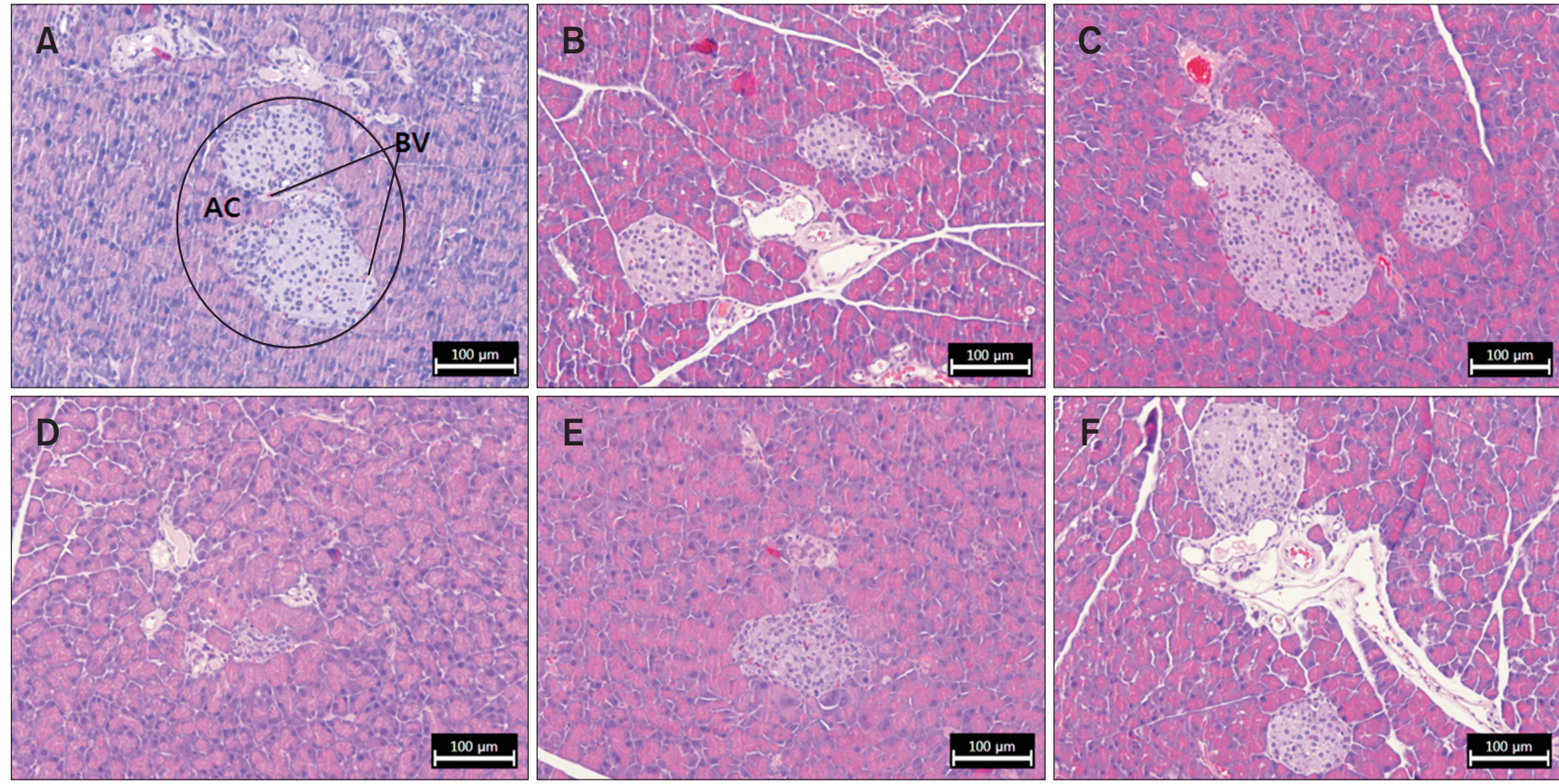

Fig. 6. Histopathologic changes of the pancreas (H\&E stain, scale bars=100 $\mu \mathrm{m}$ ). (A) In normal mouse, the pancreas architectures were well preserved. Islet of Langerhans (circle). AC, acinar cells; BV, blood vessels. (B) In sugar-Omija extract tea (SO) treated mouse, the pancreas architectures were well preserved. (C) In LuoHanGuo-Omija extract tea (LO) treated mouse, the pancreas architectures were well preserved. (D) In streptozotocin (STZ) treated mouse, the destruction of insulin-producing $\beta$-cells and reduction of Langerhans islet size were observed. (E) In STZ+SO (STZ/SO) treated mouse, the destruction of insulin-producing $\beta$-cells and reduction of Langerhans islet size were observed. (F) In STZ+LO (STZ/LO) treated mouse, the pancreas and blood vessels architectures were relatively well preserved.

LuoHanGuo has been reported to have sweeter taste than sugar (Hussain et al., 1990; Suzuki et al., 2005). It is also effective in treatment of diseases that require controlled sugar intake such as diabetes, obesity, hypertension, and heart disease (Si et al., 1996; Suzuki et al., 2005; Takeo et al., 2002). In this regard, this study was based on the idea that patients with diabetes and related diseases as well as those on a diet could safely consume foods by using LuoHanGuo extract as sugar substitute. Therefore, this study examined the effects of $\mathrm{SO}$ and $\mathrm{LO}$ to the blood glucose levels and metabolism of the STZ-induced diabetic mice. Moreover, the positive effects of LuoHanGuo extract were also proven.

In the animal experiment, STZ induced diabetes by destroying $\beta$-cell in the pancreatic islets of Langerhans and decreasing insulin secretion. Although transport and use of glucose was reduced due to decreased insulin secretion, it accelerated gluconeogenesis resulting to hyperglycemia (Goldberg, 1981). According to the measurements conducted, the blood glucose level of the STZ-treated group increased for two weeks of the experiment after STZ induction; however, the rate of blood glucose increase was significantly decreased in the STZ/ LO group from the third day of administering the Omija extract after five days of the STZ treatment as compared to the STZ group $(\mathrm{p}<0.001)$. Regarding glucose tolerance, the blood glucose was at the highest level between 30 60 minutes in general and it gradually fell between 90 and 120 minutes. Then, the value reverted back to the fasting level; however, there was a report that high blood glucose level can be maintained up to 120 minutes under insulin depletion and diabetic state, such as insulin resistance (Sosenko et al., 2008). In the IPGTTs, glucose was injected at a concentration level of $1.5 \mathrm{~g} / \mathrm{kg}$ and the blood glucose of the tail vein blood was measured 120 minutes later. According to the results, the blood glucose of the STZ/LO group significantly decreased compared to the STZ group $(\mathrm{p}<0.05)$. Based from the results of the blood glucose measurement and IPGTTs, it was confirmed that LuoHanGuo extract was effective in decreasing blood glucose level.

The weight change among the STZ treated groups, the STZ/ SO group and STZ/LO group showed similar pattern. From the third day of administering the Omija extract and after five days of the STZ treatment, the weight of STZ/LO group significantly increased as compared to that of the STZ group $(p<0.001)$. There was also a report indicating that weight tends to decrease when a person is afflicted with diabetes caused by insulin resistance owing to imbalance in energy metabolism (Jekal et al., 2008). Thus, it was determined that LuoHanGuo extract impeded weight loss caused by STZinduced diabetes.

AST, ALT, and ALP, which are the indicators of liver damage, 
are the enzymes existing in liver cells. In case of liver damage, it is secreted resulting to increased level in the blood (Bursch \& Schulte-Hermann, 1986; Lee et al., 2013; Nannipieri et al., 2005; Park et al., 2015; Steer et al., 1985). There was no significant difference in ALP among the groups and AST of STZ/SO group significantly increased as compared to that of the STZ group $(\mathrm{p}<0.05)$. Also, ALP in the STZ/LO group decreased compared to that of the STZ group and significantly decreased as compared to that of the STZ/SO group ( $\mathrm{p}<0.001)$. On the other hand, ALT of the STZ/LO group decreased as compared to that of the STZ group and it significantly decreased compared to the STZ/SO group ( $\mathrm{p}<0.001)$. It was shown that LuoHanGuo extract reduced secretion of AST and ALT that were increased by STZ; thus, it was effective in improving liver damage. It was also possible to verify that AST and ALT in the STZ/SO group increased compared to those of the STZ group and it revealed the negative effects of sugar in terms of liver function. When a patient has abnormal liver function, serum albumin and total protein decrease whereas bilirubin concentration increases (Whitney et al., 2007). There was no significant difference among the groups regarding total bilirubin, and albumin and total protein in the STZ/ LO group increased compared to the STZ group. Therefore, it was determined that LuoHanGuo extract was effective in alleviating liver damage caused by STZ.

BUN, which is the index of kidney damage, and creatinine levels increased in the blood in case of kidney damage (Allen, 2012; Bessman \& Carpenter, 1985; Coresh et al., 2001; Levey et al., 1999; Vaidya et al., 2006). Among the non-STZ treated groups, BUN of the SO group $(\mathrm{p}<0.05)$ and $\mathrm{LO}$ group $(\mathrm{p}<0.05)$ significantly decreased compared to the normal group. Among the STZ treated groups, BUN of the STZ/ LO group increased as compared to that of the STZ group and significantly increased compared to the STZ/SO group $(\mathrm{p}<0.05)$. However, there was no significant difference among the groups regarding creatinine. LuoHanGuo extract resulted to decreased BUN among the non-STZ treated groups while it caused increase among the STZ treated groups. Such result implied that it is not effective in improving kidney function, particularly for STZ-induced diabetes.

With diabetes, the speed of converting the plasma free fatty acid to triglyceride in the liver increases in order to use fat as the energy source instead of glucose and consequently, it causes increased synthesis of LDL-cholesterol (Liao et al., 2010). Moreover, it causes abnormal lipid metabolism that triggers increase of cholesterol biosynthesis in the course of accelerating LDL secretion (Hong et al., 2002). Regarding triglyceride, LDL-C, and total cholesterol of lipid metabolism, STZ/LO group showed lower values compared to the STZ group and STZ/SO group. Particularly, total cholesterol of the
STZ/LO group was significantly lower as compared to that of the STZ/SO group ( $<<0.05)$. In addition, HDL-C of the STZ/ LO group was higher as compared to that of the STZ group and significantly higher compared to the STZ/SO group $(\mathrm{p}<0.05)$. Thus, it was determined that LuoHanGuo extract was effective in improving abnormal lipid metabolism in case of diabetes.

According to the observation of the pancreatic tissues, damage in the beta-cell in the islets of Langerhans and big vacuolation were noticeably observed in the STZ group and STZ/SO group among the STZ treated groups (Fig. 6D and E). Compared to the STZ group and STZ/SO group, relatively normal beta-cell and blood vessel structure in the STZ/LO group was preserved, and there was no vacuolation observed (Fig. 6F). From the third day of administering the Omija extract after five days of the STZ treatment, the blood glucose level in the STZ/LO group significantly decreased as compared to that of the STZ group $(\mathrm{p}<0.001)$. Since such result corresponded to the measurement of blood glucose, it was determined that LuoHanGuo extract was effective in alleviating pancreatic damage caused by STZ.

Through this study, it was determined that the LO containing extract of LuoHanGuo displayed hypoglycemic effect, prevented weight loss caused by diabetes, recovered liver function, and improved lipid metabolism. In addition, such positive effects were obviously shown when compared with the experimental group treated with the SO.

\section{CONCLUSIONS}

The LO were considered as favorable foods that can be safely taken among those who are on a diet, people who need to control their sugar intake, and persons with diabetes, obesity, hypertension, and heart disease.

\section{CONFLICT OF INTEREST}

No potential conflict of interest relevant to this article was reported.

\section{ACKNOWLEDGMENTS}

This work was supported by Industry-university-research collaboration technology development support program funded by the Small and Medium Business Administration (SMBA, Korea) (C0267375); and by Basic Science Research Program through the National Research foundation of Korea (NRF) grant funded by the Ministry of Education (NRF2014R1A1A3051040). 


\section{REFERENCES}

Allen P J (2012) Creatine metabolism and psychiatric disorders: does creatine supplementation have therapeutic value? Neurosci. Biobehav. Rev. 36, 1442-1462.

Alonso S and Setser C (1994) Functional replacements for sugars in foods. Trends Food Sci. Technol. 5, 139-146.

Bessman S P and Carpenter C L (1985) The creatine-creatine phosphate energy shuttle. Annu. Rev. Biochem. 54, 831-862.

Bray G A, Nielsen S J, and Popkin B M (2004) Consumption of highfructose corn syrup in beverages may play a role in the epidemic of obesity. Am. J. Clin. Nutr. 79, 537-543.

Bursch W and Schulte-Hermann R (1986) Cytoprotective effect of the prostacyclin derivative iloprost against liver cell death induced by the hepatotoxins carbon tetracholoride and brombenzen. Klin. Wochenschr. 7, 47-50.

Chen W J, Wang J, Qi X Y, and Xie B J (2007) The antioxidant activities of natural sweeteners, mogrosides, from fruits of Siraitia grosvenori. Int. J. Food Sci. Nutr. 58, 548-556.

Coresh J, Wei G L, McQuillan G, Brancati F L, Levey A S, Jones C, and Klag M J (2001) Prevalence of high blood pressure and elevated serum creatinine level in the United States: findings from the third national health and nutrition examination survey (1988-1994). Arch. Intern. Med. 161, 1207-1216.

Goldberg R B (1981) Lipid disorders in diabetes. Diabetes Care 4, 561 572.

Haller H (1998) The clinical importance of postprandial glucose. Diabetes Res. Clin. Pract. 40, S43-S49.

Hong J H, Park M R, and Rhee S J (2002) Effects of YK-209 mulberry leaves on HMG-CoA reductase and lipid composition of liver on streptozotocin-induced diabetic rats. J. Korean Soc. Food Sci. Nutr. 31, 826-833.

Huang J (2006) Control of major diseases and pests in Siraitia grosvenorii. J. Guangxi Acad. Sci. 22, 188-192.

Hussain R A, Lin Y M, Poveda L J, Bordas E, Chung B S, Pezzuto J M, Soejarto D D, and Kinghorn A D (1990) Plant-derived sweetening agents: saccharide and polyol constituents of some sweet-tasting plants. J. Ethnopharmacol. 28, 103-151.

Institute of Beijing Botany of Academic Sinica (1975) Chinese Higher Plants (Zhongguo Gaodeng Zhiwu Tujian), pp. 359, (Chinese Academic Press, Beijing).

Jekal S J, Lee K S, Chung O B, and Lee J H (2008) Effects of electrical stimulation on wound healing and skin mast cells in streptozotocininduced diabetic rats. Korean J. Clin. Lab. Sci. 40, 118-128.

Jenkins D J, Wolever T M, and Jenkins A L (1988) Starchy foods and glycemic index. Diabetes Care 11, 149-159.

Kim J S and Choi S Y (2008) Physicochemical properties and antioxidative activities of Omija(Schizandra chinensis Bailon). Korean J. Food Nutr. 21, 35-42.

Kim S I, Sim K H, Ju S Y, and Han Y S (2009) A study of antioxidative and hypoglycemic activities of Omija(Schizandra chinensis Baillon) extract under variable extract conditions. Korean J. Food Nutr. 22, 41-47.

Korean Diabetes Association and Korea Centers for Disease Control and Prevention (2012) Diabetes fact sheet in Korea 2012, pp. 2, (Korean Diabetes Association, Seoul; Korea Centers for Disease Control and Prevention, Osong).
Kraegen E W, Clark P W, Jenkins A B, Daley E A, Chisholm D J, and Storlien L H (1991) Development of muscle insulin resistance after liver insulin resistance in high-fat-fed rats. Diabetes 40, 1397-1403.

Lebovitz H E (1998) Postprandial hyperglycaemic state: importance and consequences. Diabetes Res. Clin. Pract. 40, S27-S28.

Lee J H, Hyun S H, Park K T, Ahn T H, and Kim I S (2013) Correlation of chronic hepatitis $\mathrm{B}$ virus infection with diabetes mellitus indicators. Korean J. Clin. Lab. Sci. 45, 9-15.

Levey A S, Bosch J P, Lewis J B, Greene T, Rogers N, and Roth D (1999) A more accurate method to estimate glomerular filtration rate from serum creatinine: a new prediction equation. Ann. Intern. Med. $\mathbf{1 3 0}$ 461-470.

Li D and Zhang H (2000) Studies and uses of Chinese medicine Luohanguo a special local product of Guangxi. Guihaia 20, 270-276.

Liao Z, Chen X, and Wu M (2010) Antidiabetic effect of flavones from Cirsium japonicum DC in diabetic rats. Arch. Pharm. Res. 33, 353362.

Luo J, Rizkalla S W, Lerer-Metzger M, Boillot J, Ardeleanu A, Bruzzo F, Chevalier A, and Slama G (1995) A fructose-rich diet decreases insulin-stimulated glucose incorporation into lipids but not glucose transport in adipocytes of normal and diabetic rats. J. Nutr. 125, 164-171.

Ministry of Health and Welfare (2014) Korea health statistics 2014 Korea national health and nutrition examination surveys (KNHANES $V$-1) (Ministry of Health and Welfare, Seoul).

Nakamura J, Hamada Y, Sakakibara F, Hara T, Wakao T, Mori K, Nakashima E, Naruse K, Kamijo M, Koh N, and Hotta N (2001) Physiological and morphometric analyses of neuropathy in sucrosefed OLETF rats. Diabetes Res. Clin. Pract. 51, 9-20.

Nannipieri M, Gonzales C, Baldi S, Posadas R, Williams K, Haffner S M, Stern M P, and Ferrannini E (2005) Liver enzymes, the metabolic syndrome, and incident diabetes the Mexico city diabetes study. Diabetes Care 28, 1757-1762.

Park C J and Han J S (2015) Hypoglycemic effect of Jicama (Pachyrhizus erosus) extract on streptozotocin-induced diabetic mice. Prev. Nutr. Food Sci. 20, 88-93.

Park S M, Kim Y S, Yoon I C, Seo E H, Ko B S, and Choi S B (2002) Development and hypoglycemic effect of low-fat and sugar free cookie. Korean J. Food Sci. Technol. 34, 487-492.

Park S Y, Pak S J, Kang S J, Kim N Y, Kim D S, and Kim M J (2015) Effects of the C3G/D3G anthocyanins-rich black soybean testa extracts on improvement of lipid profiles in STZ-induced diabetic rats. J. Nutr. Health 48, 299-309.

Shin H L (2010) Consuver attitude survey: beverage purchasing behaviors and preference. MS thesis, (Sejong University, Seoul).

Si J, Chen D, Chang Q, and Shen L (1996) Isolation and determination of cucurbitane-glycosides from fresh fruits of Siraitia grosvenorii. Acta Botanica Sinica 38, 489-494.

Soedamah-Muthu S S, Chaturvedi N, Schalkwijk C G, Stehouwer C D A, Ebeling P, and Fuller J H; EURODIAB Prospective Complications Study Group (2006) Soluble vascular cell adhesion molecule-1 and soluble E-selectin are associated with micro-and macrovascular complications in type 1 diabetic patients. J. Diabetes Complicat. 20, 188-195. 
Sosenko J M, Palmer J P, Rafkin-Mervis L, Krischer J P, Cuthbertson D, Matheson D, and Skyler J S (2008) Glucose and C-peptide changes in the perionset period of type 1 diabetes in the diabetes prevention trial-type 1. Diabetes Care 31, 2188-2192.

Steer K A, Sochor M, and McLean P (1985) Renal hypertrophy in experimental diabetes: Changes in pentose phosphate pathway activity. Diabetes $\mathbf{3 4}, \mathbf{4 8 5}-490$.

Steven E K, Rebecca L H, and Kristina M U (2006) Mechanism linking obesity to insulin resistance and type 2 diabetes. Nature 444, 840846.

Storlien L H, Jenkins A B, Chisholm D J, Pascoe W S, Khouri S, and Kraegen E W (1991) Influence of dietary fat composition on development of insulin resistance in rats. Relationship to muscle triglyceride and omega-3 fatty acids in muscle phospholipid. Diabetes 40, 280-289.

Suzuki Y A, Murata Y, Inui H, Sugiura M, and Nakano Y (2005) Triterpene glycosides of Siraitia grosvenori inhibit rat intestinal maltase and suppress the rise in blood glucose level after a single oral administration of maltose in rats. J. Agric. Food Chem. 53, 29412946.

Takasaki M, Konoshima T, Murata Y, Sugiura M, Nishino H, and Tokuda H (2003) Anti carcinogenic activity of natural sweeteners, cucurbitane glycosides, from Momordica grosvenori. Cancer Lett. 198, 37-42.

Takeo E, Yoshida H, Tada N, Shingu T, Matsuura H, and Murata Y (2002) Sweet elements of Siraitia (Momordica) grosvenori inhibit oxidative modification of low density lipoprotein. J. Atheroscler. Thromb. 9, 114-120.

Tang S, Bin X, Peng Y, Zhou Y, Wang L, and Zhong Y (2007) Assessment of genetic diversity in cultivars and wild accessions of Luohanguo (Siraitia grosvenorii [Swingle]), a species with edible and medicinal sweet fruits endemic to southern China, using RAPD and AFLP markers. Genet. Resour. Crop Evolut. 54, 1053-1061.
Ukiya M, Akihisa T, Tokuda H, Toriumi M, Mukainaka T, and Banno $N$ (2002) Inhibitory effects of cucurbitane glycosides and other triterpenoids from the fruit of Momordica grosvenori on Epstein-Barrvirus early antigen induced by tumor promoter 12-Otetradecanoylphorbol-13-acetate. J. Agric. Food Chem. 50, 6710-6715.

Ukiya M, Akihisa T, Yasukawa K, Tokuda H, Toriumi M, and Koike K (2002) Anti-inflammatory and anti-tumor promoting effects of cucurbitane glycosides from the roots of Bryonia dioica. J. Nat. Prod. 65, 179183.

Vaidya V S, Ramirez V, Ichimura T, Bobadilla N A, and Bonventre J V (2006) Urinary kidney injury molecule-1: a sensitive quantitative biomarker for early detection of kidney tubular injury. Am. J. Physiol. Renal Physiol. 290, F517-F529.

West K M, Ahuja M S, and Bennett P H (1983) The role of circulating glucose and triglyceride concentrations and their interaction with other "risk factors" as determinants of arterial disease in nine diabetic population samples from the WHO multi-national study. Diabetes Care 6, 361-369.

Whitney E, Debruyne L K, Pinna K, and Rolfes S R (2007) Nutrition for Health Health Care, 4th ed. (Thomson, New York).

Yoshikawa S, Murata Y, Sugiura M, Kiso T, Shizuma M, Kitahata S, and Nakano H (2005) Transglycosylation of mogroside V, a triterpene glycoside in Siraitia grosvenori by cyclodextrin glucanotransferase and improvement of the qualities of sweetness. J. Appl. Glycosci. 52, 247-252.

Zhou J and Tang S (2006) Genetic diversity of cultivated Luohanguo (Siraitia grosvenorii) revealed by RAPD markers. Mol. Plant Breed. 4, 71-78. 